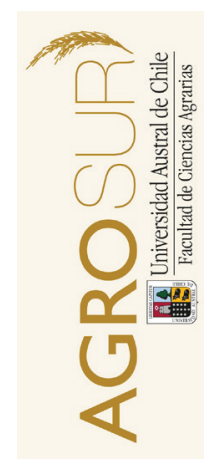

\title{
Variación de la biodisponibilidad de los herbicidas metsulfuron - metil y triasulfuron bajo distintas condiciones de encalado en dos suelos volcánicos del sur de Chile
}

\author{
Variation of the bioavailability of metsulfuron-methyl herbicides and triasulfuron \\ under different liming conditions in two volcanic soils of southern Chile
}

\author{
Fuentes, R. ${ }^{a *}$, Henríquez, J. ${ }^{a}$, Pinochet, $\mathrm{D}^{b}$, Clunes, J. ${ }^{b}$ \\ ${ }^{a}$ Instituto de Producción y Sanidad Vegetal, Universidad Austral de Chile. Casilla 567, Valdivia, Chile. \\ ${ }^{b}$ Instituto de Ingeniería Agraria y Suelos, Universidad Austral de Chile, Casilla 567, Valdivia, Chile.
}

\begin{abstract}
ARTICLE INFO
Keywords:

Metsulfuron-methyl

Triasulfuron

Liming

Soil adsorption

Original Research Article

Soil Science

*Corresponding author:

Ricardo Fuentes

E-mail address:

rfuentes@uach.cl

\section{A B S T R A C T}

The use of sulfonylurea herbicides together with calcareous amendments is a common practice in acid soils derived from volcanic ash, especially in cereal crops. The objective of this study was to determine, through bioassays, the bioavailability of metsulfuron-methyl and triasulfuron herbicides by liming in two soils derived from volcanic ash. The soils used were a Hapludand series Malihue and a Paleohumult series Fresia. The indicator species was pea (Pisum sativum L.), planted in pots with both soils and under four levels of liming (equivalent to $0,1.9,3.7$ and $7.4 \mathrm{t} \mathrm{ha}^{-1}$ of $\mathrm{Ca}(\mathrm{OH})_{2}$ ) and 11 concentrations of herbicides. In each bioassay a control treatment with non-adsorptive substrate with non-limestone quartz was included. After 8 days of herbicide application, the radical length of the indicator species was measured and the values obtained were adjusted to a non-linear model, the dose that inhibited $50 \%$ of the radical development $\left(\mathrm{ID}_{50}\right)$ in each treatment was determined. The bioavailability of each herbicide corresponded to the quotient between the $\mathrm{ID}_{50}$ obtained in the quartz substrate and the $\mathrm{ID}_{50}$ obtained in each treatment. The results showed that the adsorption of metsulfuron-methyl and triasulfuron decreased as the liming dose increased, increasing their relative bioavailability in both soils. The adsorption of triasulfuron with increasing lime applications was lower than that of metsulfuron-methyl in quantitative terms, but higher in relative terms. These results were corroborated when examining the adsorption coefficients (Kd).
\end{abstract}

\section{RESUMEN}

El uso conjunto de herbicidas sulfonilureas con enmiendas calcáreas es una práctica habitual en suelos ácidos derivados de cenizas volcánicas, especialmente en cultivos de cereales. El objetivo de este estudio fue determinar, a través de bioensayos, la biodisponiblidad de los herbicidas metsulfuron-metil y triasulfuron por efecto del encalado en dos suelos derivados de cenizas volcánicas. Los suelos utilizados fueron un Hapludand serie Malihue y un Paleohumult serie Fresia. La especie indicadora fue arveja (Pisum sativum L.), sembrada en macetas con ambos suelos y bajo cuatro niveles de encalado (equivalente a $0 ; 1,9 ; 3,7$ y 7,4 t ha ${ }^{-1}$ de $\mathrm{Ca}(\mathrm{OH})_{2}$ ) y 11 concentraciones de herbicidas. En cada bioensayo se incluyó un tratamiento testigo con sustrato no adsortivo con cuarzo sin encalado. A los 8 días de aplicados los herbicidas se midió el largo radical de la especie indicadora y los valores obtenidos fueron ajustados a un modelo no-lineal, determinándose la dosis que inhibió el 50\% del desarrollo radical $\left(\mathrm{IC}_{50}\right.$ ) en cada tratamiento. La biodisponibilidad de cada herbicida correspondió al cuociente entre el $\mathrm{IC}_{50}$ obtenido en el sustrato cuarzo y el IC ${ }_{50}$ obtenido en cada tratamiento. Los resultados demostraron que la adsorción de metsulfuron-metil y de triasulfuron disminuyó a medida que aumentó la dosis de encalado, aumentando su biodisponibilidad relativa en ambos suelos. La adsorción de triasulfuron con el incremento de las aplicaciones de cal fue inferior a la de metsulfuron-metil en términos cuantitativos, pero mayor en términos relativos. Estos resultados se corroboraron al examinar los coeficientes de adsorción $(\mathrm{Kd})$.

Palabras clave: metsulfuron-metil, triasulfuron, encalado, adsorción del suelo.

\section{INTRODUCCIÓN}

Las sulfonilureas son un grupo de herbicidas utilizados en diversos países para el control de malezas anuales en cultivos de cereales (Walker y Welch, 1989), caracterizados por presentar un amplio espectro de acción a muy bajas dosis, una alta especificidad sobre plantas y una muy baja toxicidad y bioacumulación en organismos vivos (Brown, 1990; Singh y Kulshrestha, 2006). Las sulfonilureas presentan una muy alta actividad herbicida en el suelo y después de una aplicación foliar, siendo absorbidas tanto por el follaje como por el sistema radical, para luego traslocarse bidireccionalmente dentro del floema e inhibir el metabolismo de la 
planta (Sarmah y Sabadie, 2002; Anderson et al., 2010). El mecanismo de acción de estos herbicidas consiste en la reducción en la célula, de los niveles de valina, leucina e isoleucina producto de la inhibición de la acetolactato sintasa (ALS), enzima esencial para la biosíntesis de los aminoácidos de cadena ramificada (HernándezSevillano et al., 2001). Las condiciones de humedad del suelo favorecen la acción de estos productos, los cuales son aplicados generalmente de post-emergencia, siendo altamente activos en el suelo a pesar de ser utilizados en bajas dosis (Fuentes et al., 2005). La efectividad de herbicidas como el metsulfuron-metil y triasulfuron depende de las condiciones climáticas y de la disponibilidad que estos presentan en el suelo para ser absorbidos por las plantas. Esta disponibilidad está influenciada principalmente por procesos de adsorción-desorción, movilidad y degradación de las sulfonilureas en el suelo (Sekutowski, 2011).

La adsorción de moléculas de herbicidas desde la solución del suelo por los coloides orgánicos e inorgánicos, que presentan en su superficie cargas asociadas a los grupos hidroxilos que son dependientes del $\mathrm{pH}$ (Rahman et al., 1996), produce una disminución de la concentración y disponibilidad del producto en la solución del suelo (Abdullah et al., 2001), siendo descrita como un importante factor de la disipación biótica y abiótica de los herbicidas en el suelo (Blacklow y Pheloung, 1992). Las sulfonilureas presentan una carga electrostática negativa debido a su desprotonización como ácidos débiles, dando lugar a que su presencia en la solución del suelo sea altamente dependiente del pH y el contenido de materia orgánica (Ismail y Ooi, 2012). De esta manera, habrá mayor adsorción de sulfonilureas en suelos con $\mathrm{pH}$ más ácidos y mayor contenido de MO (Ren et al., 2011). Por el contrario, al modificar el pH del suelo cercano a la neutralidad, existirá un aumento de la capacidad de intercambio de cationes y dado la carga variable del suelo, se producirá una mayor repulsión de las moléculas cargadas negativamente. Lo anterior implica que habrá una mayor predominancia de la forma aniónica del herbicida, que soluble en agua, aumentando su potencial lixiviación debido a una menor sorción sobre las superficie cargadas de los coloides del suelo (Cáceres et al., 2010b).

Por otra parte, una práctica agrícola habitual en suelos ácidos, como los Andisoles, es el uso de enmiendas calcáreas para modificar el $\mathrm{pH}$ del suelo y favorecer las condiciones de desarrollo de los cultivos. La aplicación de cal en suelos ácidos al incrementar el pH causa un aumento de la carga negativa del suelo (Mora y Demanet, 1999) y con ello una mayor capacidad para repeler herbicidas aniónicos como las sulfonilureas, pudiendo aumentar su biodisponibilidad en la solución suelo. El objetivo de este trabajo fue determinar la variabilidad de la biodisponibilidad de los herbicidas triasulfuron y metsulfuron - metil, frente al cambio del $\mathrm{pH}$ con distintas dosis de encalado en dos suelos Andisol bajo condiciones controladas.

\section{MATERIAL Y MÉTODO}

Este estudio fue realizado en Laboratorio de Malherbología de la Universidad Austral de Chile. Las muestras de suelo fueron colectadas a $10 \mathrm{~cm}$ de profundidad desde la Serie Malihue clasificada como Hapludand y la Serie Fresia clasificada como Palehumult (Luzio y Casanova, 2006). Las muestras de ambos suelos fueron secadas a $35^{\circ} \mathrm{C}$ durante 7 días, tamizadas a $4 \mathrm{~mm}$ y esterilizadas con bromuro de metilo por 48 horas. Luego se realizó la caracterización química y física de los suelos (Cuadro 1).

Ambos suelos fueron separados en cuatro bolsas de polietileno de $10 \mathrm{~kg}$ cada una aproximadamente y se les aplicó hidróxido de calcio $\left(\mathrm{Ca}(\mathrm{OH})_{2}\right)$ como enmienda calcárea en 4 tratamientos, un control sin aplicación y 3 dosis crecientes de $\left(\mathrm{Ca}(\mathrm{OH})_{2}\right)$ de 1,9; 3,7; $7,4 \mathrm{t} \mathrm{ha}^{-1}$. Estas muestras fueron incubadas a $35^{\circ} \mathrm{C} \mathrm{du}$ rante 48 horas, con una humedad inicial de un $10 \%$ bss. Al término de la incubación se midió el pH de cada tratamiento aplicado. Con las muestras de suelo en

Cuadro 1: Características químicas y físicas de los suelos Malihue y Fresia utilizados en el estudio.

Table 1: Chemical and physical characteristics of the Malihue and Fresia soil used in this study.

\begin{tabular}{|c|c|c|}
\hline Características & $\begin{array}{c}\text { Suelo } \\
\text { Malihue }\end{array}$ & $\begin{array}{l}\text { Suelo } \\
\text { Fresia }\end{array}$ \\
\hline Materia orgánica $\left(\mathrm{g} 100 \mathrm{~g}^{-1}\right.$ ) & 16 & 11 \\
\hline Fósforo Olsen (mg kg-1) & 2,0 & 3,2 \\
\hline pH (agua) & 5,22 & 5,55 \\
\hline $\mathrm{Ca}\left(\mathrm{cmol}_{+} \mathrm{kg}^{-1}\right)$ & 2,191 & 1,940 \\
\hline $\mathrm{Mg}\left(\mathrm{cmol}_{+} \mathrm{kg}^{-1}\right)$ & 0,728 & 0,252 \\
\hline $\mathrm{K}\left(\mathrm{cmol}_{+} \mathrm{kg}^{-1}\right)$ & 0,235 & 0,066 \\
\hline $\mathrm{Na}\left(\mathrm{cmol}_{+} \mathrm{kg}^{-1}\right)$ & 0,10 & 0,046 \\
\hline $\begin{array}{l}\text { Aluminio Intercambiable } \\
\left(\mathrm{cmol}_{+} \mathrm{kg}^{-1}\right)\end{array}$ & 0,115 & 1,723 \\
\hline Arena $\left(\mathrm{g} 100 \mathrm{~g}^{-1}\right)$ & 10 & 4.4 \\
\hline Limo (g $100 \mathrm{~g}^{-1}$ ) & 62,1 & 32,1 \\
\hline Arcilla (g $100 \mathrm{~g}^{-1}$ ) & 27,9 & 63,5 \\
\hline Densidad aparente $\left(\mathrm{g} \mathrm{cm}^{-3}\right)$ & 0,65 & 0,72 \\
\hline $\begin{array}{l}\text { Capacidad máxima de retención } \\
\left({\left.\mathrm{g} 100 \mathrm{~g}^{-1}\right)}\right.\end{array}$ & 122 & 78 \\
\hline
\end{tabular}


distintos niveles de $\mathrm{pH}$ se subdividieron en unidad experimentales de $150 \mathrm{~g}$ de suelo en vasos plásticos de $300 \mathrm{ml}$, en los cuales se sembraron 5 semillas de arveja (Pisum sativum cv. Calle Calle), como especie indicado$\mathrm{ra}$, a una profundidad de $15 \mathrm{~mm}$.

Se utilizaron 2 herbicidas del grupo de las sulfonilureas en este experimento, Metsulfuron-metil y Triasulfuron. Ambos herbicidas fueron aplicados en 10 dosis crecientes e iguales para cada suelo. Para el suelo Serie Malihue las dosis fueron de 0,008; 0,02; 0,$07 ; 0,22 ; 0,66 ; 1,97 ; 5,92 ; 17,76 ; 53,29 ; 159,88 \mathrm{mg}$ ia kg suelo seco $^{-1}$ y para el suelo Serie Fresia fueron de 0,$012 ; 0,036 ; 0,109 ; 0,328 ; 0,98 ; 2,95 ; 8,85 ; 26,57$; 79,$71 ; 239,13$ mg ia kg suelo seco-1 ${ }^{-1}$ suelo seco. Como testigo o sustrato inerte se utilizó arena de cuarzo tamizado a $2 \mathrm{~mm}$, debido a que no presenta carga variable se asume que no tiene capacidad de adsorción y por lo tanto, que el $100 \%$ de la dosis aplicada se encontraría disponible en la solución del suelo. Tanto las dosis de herbicidas como el control fueron consideras con cinco repeticiones cada una. Finalmente, cada vaso de $300 \mathrm{ml}$ fue incubado con humedad de $90 \%$ de la capacidad máxima de retención, en dos cámaras bioclimáticas en ausencia de luz y a una temperatura constante de $25^{\circ} \mathrm{C}$ por 8 días, momento en el cual se determinó el largo radical de la especie indicadora en cada una de las unidades experimentales.

\section{Determinación de la adsorción de los herbicidas en los suelos}

Para la determinación de la adsorción se utilizó el modelo de bioensayo descrito por Stork y Hannah (1996), quienes usaron la variación del largo radical de una especie vegetal como un indicador biológico de la respuesta a dosis crecientes de herbicida. El conjunto de datos fue ajustados a curvas logísticas, con la cual se determinó la dosis de herbicida que inhibe el 50\% del crecimiento radical de arveja (P. sativum L.) en los distintos tratamientos con encalado $\left(\mathrm{IC}_{50}\right)$ y se comparó con el valor de $\mathrm{IC}_{50}$ del sustrato inerte de cuarzo. Los supuestos considerados en el uso del testigo cuarzo fueron que este sustrato era inerte, no presentaría adsorción, no tendría una degradación biológica significativa y que la disipación del herbicida es insignificante durante el periodo de evaluación. De esa manera, se asumió que la diferencia entre los $\mathrm{IC}_{50}$ del testigo y los IC ${ }_{50}$ de los tratamientos, expresada en ng ia kgs.s. ${ }^{-1}$, representarían la cantidad de herbicida adsorbido por el suelo en cada una de las condiciones de encalado y por ende sería una estimación del porcentaje de herbicida biodisponible en solución suelo. Además, se estimó el coeficiente de adsorción (Kd), calculando la cantidad adsorbida por unidad de peso de suelo seco (ng ia kgs.s. ${ }^{-1}$ ), dividido por la concentración de la solución (ng ia $\mathrm{L}^{-1}$ ), descrito por Walker et al. (1989).
La cantidad adsorbida se obtiene de la diferencia entre el IC $\mathrm{I}_{50}$ del testigo con el $\mathrm{IC}_{50}$ del tratamiento.

\section{Análisis estadístico}

Cada bioensayo se manejó en un diseño experimental completamente al azar con tratamientos dispuestos en un arreglo factorial correspondiente a dos tipos de herbicidas por cuatro niveles de enmienda calcárea por 11 dosis de herbicidas con cinco repeticiones. En cada bioensayo se agregó un testigo cuarzo para cada herbicida. Los valores de largo radical de cada tratamiento fueron ajustados a la siguiente función logística descrita por Stork y Hannah (1996):

$$
y=a+c /\left(1+e^{b(x-m)}\right)
$$

Donde; $\mathbf{y}$ es el largo radical relativo del bioensayo; $\mathbf{a}+\mathbf{c}$ y a son los coeficientes de las mayores y menores asíntotas de la curva, respectivamente; $\mathbf{b}$ es el coeficiente de la gradiente de la curva, $\mathbf{m}=\ln \left(\mathrm{IC}_{50}+1\right)$ es el punto de inflexión horizontal a mitad de camino entre $\mathbf{a}+\mathbf{c}$ y $\mathbf{a}, \mathrm{y} \mathbf{x}$ es la transformación logarítmica de la concentración de herbicida aplicada. De esa manera, usando el programa GRAPHPAD PRISM 4.0 se ajustaron las regresiones y se determinó la dosis que inhibieron el $50 \%$ del crecimiento radical de la planta indicadora $\left(\mathrm{IC}_{50}\right)$ en los distintos tratamientos. Luego de obtenidos las distintos $\mathrm{IC}_{50}$ por repetición se les aplicó un análisis de varianza multifactorial con el programa STATGRAPHIC 2.0 y se determinaron diferencias significativas entre los valores medios de $\mathrm{IC}_{50}$ de los distintos tratamientos (Tukey 5\%).

\section{RESULTADOS}

\section{Suelo Malihue}

El pH observado en el suelo Malihue después de aplicado las dosis equivalentes a $0,1,9,3,7$ y 7,4 t ha $^{-1}$ de $\mathrm{Ca}(\mathrm{OH})_{2}$ fue de $5,22,5,49,5,72$ y 6,18 , respectivamente. Los valores obtenidos indicarían que para aumentar el pH de este suelo en 0,1 unidades se necesitarían un equivalente de de $\mathrm{Ca}(\mathrm{OH})_{2}$ entre 0,70 a $0,77 \mathrm{t} \mathrm{ha}^{-1}$.

En el Cuadro 2 se presentan los coeficientes de regresión y los coeficientes de determinación obtenidos al ajustar los valores observados de largo radical por efecto de las diferentes dosis de metsulfuron-etil y triasulfuron al modelo descrito por Stork y Hannah (1996) en el suelo Malihue. Los coeficientes de determinación obtenidos fueron superiores a 0,96 , confirmando que este modelo permite una buena estimación de la relación entre la variación del largo radical y la dosis de ambos herbicidas utilizados.

El valor de $\mathrm{IC}_{50}$ mas bajo obtenido en el tramiento control (cuarzo), comparado con los tratamientos de suelo tratados con cal, estaría validando el supuesto 
que en este sustrato no existiría ningún tipo de adsorción de herbicida, ya que es un material inerte (sin reactividad), por tanto la dosis de herbicida aplicada se encontraría al 100\% disponible en la solución suelo. $\mathrm{Al}$ analizar gráficamente la respuesta del largo radical a las diferentes dosis (Figura 1 y 2) se observa que, tanto en metsulfuron-metil como en triasulfuron, el efecto inhibitorio del crecimiento radical de la arveja se logra con menor dosis de herbicida en el tratamiento con cuarzo respecto a los tratamientos con suelo, requiriéndose en estos últimos mayores dosis de producto para lograr el mismo efecto en la medida que se reducen las dosis de cal.

$\mathrm{Al}$ comparar estadísticamente los valores de $\mathrm{IC}_{50} \mathrm{ob}-$ tenidos entre las diferentes dosis de encalado y el sustrato de cuarzo (Cuadro 3) se observa que, tanto para

Cuadro 2: Coeficientes de regresión y determinación del modelo de curvas de respuesta utilizados para metsulfuron-metil y triasulfuron bajo distintas dosis de encalado en el suelo Malihue.

Table 2: Regression and determination coefficients of the response curve model used for metsulfuron-methyl and triasulfuron under different doses of liming in Malihue soil.

\begin{tabular}{|c|c|c|c|c|c|c|}
\hline \multirow[b]{2}{*}{ Herbicida } & \multirow[b]{2}{*}{ Coeficientes $(*)$} & \multicolumn{5}{|c|}{ Dosis de $\mathrm{Ca}(\mathrm{OH})^{2}$ t ha-1 } \\
\hline & & $\mathbf{0}$ & 1,9 & 3,7 & 7,4 & Cuarzo \\
\hline \multirow{5}{*}{ Metsufuron-metil } & $\mathrm{m}\left(\mathrm{IC}_{50}\right)$ & 10,13 & 9,16 & 8,58 & 8,17 & 7,29 \\
\hline & a & 31,85 & 19,49 & 29,03 & 21,72 & 12,67 \\
\hline & c & 68,15 & 80,51 & 70,97 & 78,28 & 87,33 \\
\hline & $\mathrm{b}$ & 0,46 & 1,01 & 0,84 & 0,61 & 0,61 \\
\hline & $r^{2}$ & 0,97 & 0,98 & 0,96 & 0,98 & 0,99 \\
\hline \multirow{5}{*}{ Triasulfuron } & $\mathrm{m}\left(\mathrm{IC}_{50}\right)$ & 9,51 & 9,27 & 8,91 & 7,91 & 5,68 \\
\hline & a & 23,92 & 20,83 & 17,66 & 18,17 & 12,63 \\
\hline & c & 76,08 & 79,17 & 82,34 & 81,83 & 87,37 \\
\hline & $\mathrm{b}$ & 0,89 & 0,75 & 0,55 & 0,49 & 0,71 \\
\hline & $r^{2}$ & 0,99 & 0,98 & 0,97 & 0,98 & 0,99 \\
\hline
\end{tabular}

${ }^{*} \mathrm{r}^{2}$ : coeficiente de determinación; $\mathrm{IC}_{50}$ : Concentración de herbicida que inhibe el $50 \%$ del crecimiento radical.

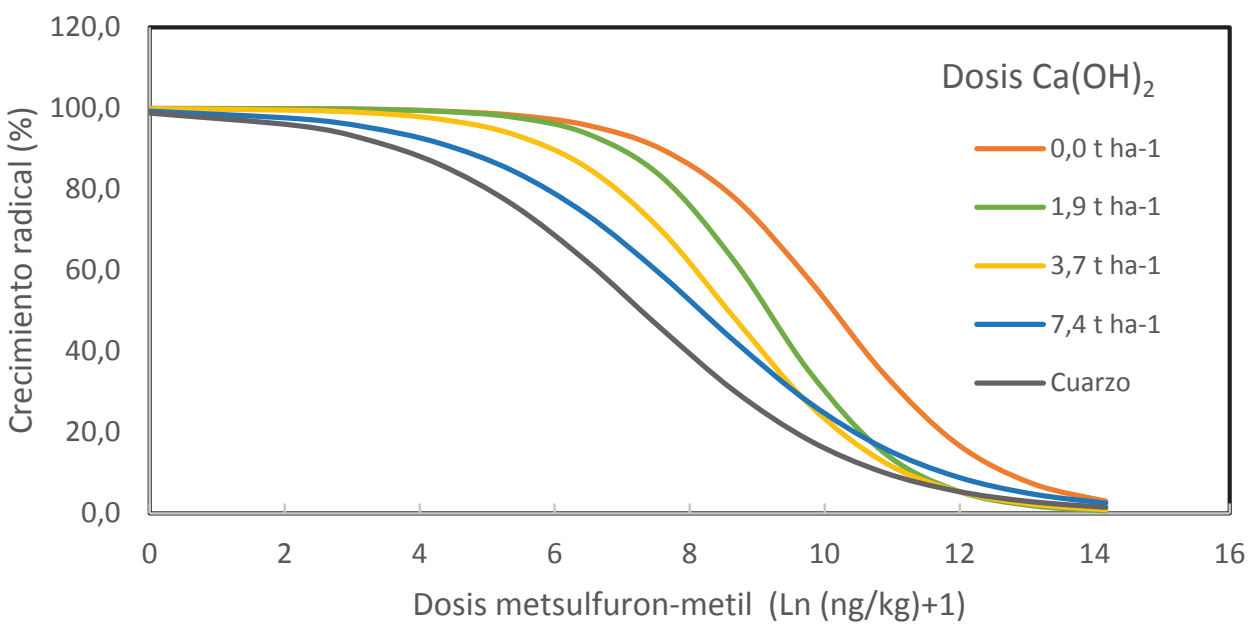

Figura 1: Curva respuesta para metsulfuron-metil bajo diferentes dosis de $\mathrm{Ca}(\mathrm{OH})_{2}$ en cuarzo y en el suelo Malihue.

Figure 1: Response curves for metsulfuron-methyl under different doses of $\mathrm{Ca}(\mathrm{OH})_{2}$ in quartz and in Malihue soil. 
metsulfuron-metil como para triasulfuron, todos los valores registrados en el sustrato cuarzo fueron significativamente inferiores comparados con los tratamientos del suelo tratados con cal. Por otra parte, los valores de $\mathrm{IC}_{50}$ en los tratamientos con suelo disminuyeron significativamente con el aumento de la dosis de $\mathrm{Ca}(\mathrm{OH})_{2}$ y todos fueron superiores al $\mathrm{IC}_{50}$ obtenidos en el cuarzo. Las dosis que inhibieron el $50 \%$ del crecimiento radical variaron según la dosis de cal aplicada al suelo, verficándose que a mayor dosis de encalado, se necesitaría la aplicación de una menor dosis de herbicida en el suelo para lograr el mismo efecto inhibitorio. De esta manera, se observó que el herbicida metsulfuron-metil necesitó en el suelo sin encalado de una dosis muy alta (25.134 ng ia $\mathrm{kg}^{-1}$ ) para lograr el mismo efecto causado con una dosis de 3.544 (ng ia $\mathrm{kg}^{-1}$ ) conseguido con un encalado de $7,4 \mathrm{t} \mathrm{ha}^{-1}$ de $\mathrm{Ca}(\mathrm{OH})_{2}$. Al expresar la dosis de $\mathrm{IC}_{50}$ en una dosis equivalente en una hectárea de suelo con una profundidad de $10 \mathrm{~cm}$ y una densidad aparente de 0,6 g $\mathrm{cm}^{-3}$ para el suelo Malihue (Cuadro 3), se puede observar que una dosis de $15 \mathrm{~g} \mathrm{ha}^{-1}$ inhibiría el 50\% del crecimiento radicular de la especie usada como indicadora, sin embargo al aplicar una dosis de encalado de 7,4 tha ${ }^{-1}$ de $\mathrm{Ca}(\mathrm{OH})_{2}$ la dosis se reduciría a $2 \mathrm{~g} \mathrm{ha}^{-1}$.

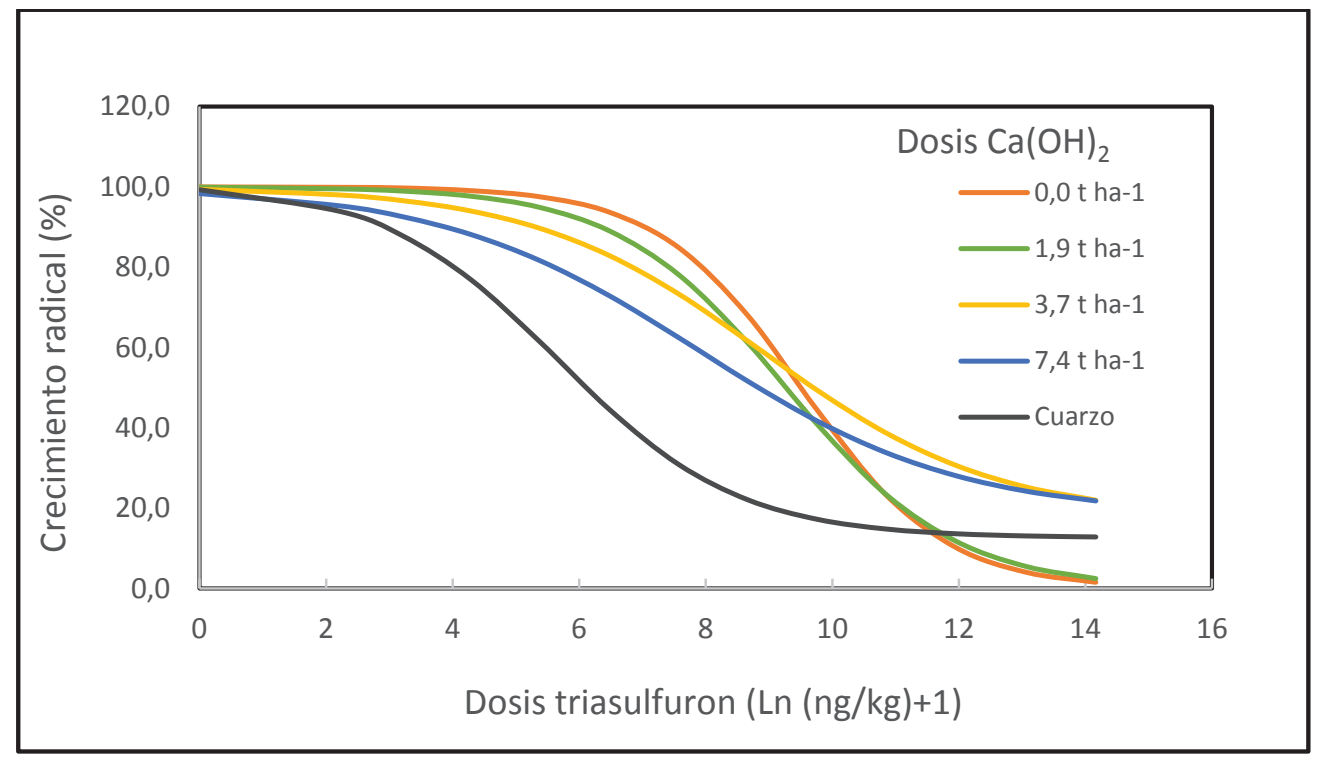

Figura 2: Curva respuesta para triasulfuron bajo diferentes dosis de $\mathrm{Ca}(\mathrm{OH})_{2}$ en cuarzo y en el suelo Malihue.

Figure 2: Response curves for metsulfuron under different doses of $\mathrm{Ca}(\mathrm{OH})_{2}$ in quartz and in Malihue soil.

Cuadro 3: Valores del $\mathrm{IC}_{50}$ y de concentración de metsulfuron-metil y triasulfuron con diferentes dosis de encalado en el suelo Malihue.

Table 3: $\quad \mathrm{IC}_{50}$ and concentration values of metsulfuron-methyl and triasulfuron with different doses of liming in Malihue soil.

\begin{tabular}{|c|c|c|c|c|c|}
\hline \multirow[b]{2}{*}{ Herbicida (unidad) } & \multicolumn{5}{|c|}{ Dosis de $\mathrm{Ca}(\mathrm{OH})_{2}\left(\mathrm{t} \mathrm{ha}^{-1}\right)$} \\
\hline & $\mathbf{0}$ & 1,9 & 3,7 & 7,4 & Cuarzo \\
\hline \multicolumn{6}{|l|}{ Metsulfuron-metil IC ${ }_{50}$} \\
\hline$\left(\operatorname{Ln}\left(\mathrm{ng} \mathrm{kg}^{-1}+1\right)\right)$ & $10,13 \mathbf{a}^{*}$ & $9,17 \mathbf{b}$ & $8,59 \mathrm{c}$ & $8,17 \mathbf{d}$ & $7,29 \mathbf{e}$ \\
\hline (ng kgs.s. ${ }^{-1}$ ) & 25.134 & 9.586 & 5.353 & 3.544 & 1.469 \\
\hline$\left(\mathrm{g} \mathrm{ha}^{-1}\right)$ & 15 & 6 & 3 & 2 & 1 \\
\hline \multicolumn{6}{|l|}{ Triasulfuron $\mathrm{IC}_{50}$} \\
\hline$\left(\operatorname{Ln}\left(\mathrm{ng} \mathrm{kg}^{-1}+1\right)\right)$ & $9,51 \mathbf{a}$ & $9,27 \mathbf{b}$ & $8,91 \mathrm{c}$ & $7,91 \mathbf{d}$ & $5,68 \mathbf{e}$ \\
\hline (ng kgs.s. ${ }^{-1}$ ) & 13.520 & 10.708 & 7.414 & 2.732 & 292 \\
\hline$\left(\mathrm{g} \mathrm{ha}^{-1}\right)$ & 8 & 6 & 4 & 2 & 0,2 \\
\hline
\end{tabular}

* = Letras diferentes en la fila indican diferencias significativas $(\mathrm{p}<0,05)$ por el test de Tukey. 
Por otra parte, considerando la diferencia entre los valores de concentración equivalente de $\mathrm{IC}_{50}$ en el tratamiento con encalado menos el $\mathrm{IC}_{50}$ del tratamiento testigo cuarzo (Cuadro 3), es posible determinar la cantidad de herbicida adsorbido. De esta forma se determinó que cuantitativamente la adsorción de metsulfuron-metil en el tratamiento sin cal $(\mathrm{pH}=5,22)$ fue máxima, alcanzando un total de $23.665 \mathrm{ng} \mathrm{kg}^{-1}$ para luego decrecer bruscamente con la aplicación de 7,4t $\mathrm{ha}^{-1}$ de cal $(\mathrm{pH}=6,18)$ hasta presentar una adsorción equivalente a $2.075 \mathrm{ng} \mathrm{kg}^{-1}$, significando una reducción de adsorción relativa de 94,2 a 58,5\%. En cuanto a la adsorción de triasulfuron, esta fue inferior a metsulfuron-metil en términos cuantitativos, presentando valores que variaron de $13.428 \mathrm{ng} \mathrm{kg}^{-1}$ en el tratamiento testigo cuarzo a $2.440 \mathrm{ng} \mathrm{kg}^{-1}$ en el tratamiento con la máxima dosis de cal, pero mayor en términos relativos, dado que la adsorción se redujo de $99,3 \%$ a $89,3 \%$, respectivamente en los mismos tratamientos de encalado. Los porcentajes de adsorción expresados en forma inversa, en términos de biodisponibilidad relativa o porcentaje de herbicida disponible en la solución suelo, se presentan en la Figura 3. En estos resultados se observa que la biodisponibilidad relativa de metsulfuronmetil existente en el tratamiento sin aplicación de cal se incrementó desde un 5,8 \% hasta un 15,3\%, 27,4\% y $41,5 \%$ con las aplicaciones de cal equivalentes a 1,9 , 3,7 y 7,4 t ha $^{-1}$ de $\mathrm{Ca}(\mathrm{OH})_{2}$, respectivamente. En el caso del triasulfuron, en general, la biodisponibilidad relativa fue menor que para metsulfuron-metil, aumentando de $2,2 \%$ en el tratamiento sin encalado a $10,7 \%$ con la dosis más alta de aplicación de cal. Estos resultados se validan al examinar los coeficientes de adsorción (Kd) presentados en el Cuadro 4, en que se observa que a medida que aumentó la dosis de encalado disminuyó el valor del Kd, variando entre 0,88 a 0,08 en metsulfuron -metil y entre 2,89 a 0,53 para triasulfuron.

\section{Suelo Fresia}

Los valores de $\mathrm{pH}$ obtenidos en este suelo como producto de las las aplicaciones de $0,1,9,3,7$ y $7,4 \mathrm{t}$ de cal ha- ${ }^{-1}$ fueron $5,55,6,17,6,66$ y 7,22, respectivamente. El requerimiento de cal para elevar el valor de $\mathrm{pH}$ es este suelo fue menor que en el suelo Malihue, necesitándose solo entre 0,31 a $0,44 \mathrm{t} \mathrm{cal} \mathrm{ha}^{-1}$ para aumentar en 0,1 unidades el pH del suelo.

Los coeficientes de regresión y de determinación obtenidos al ajustar los valores observados al mode-

Cuadro 4: Valores de Kd para metsulfuron - metil y triasulfuron por efecto de diferentes dosis de encalado con $\mathrm{Ca}(\mathrm{OH})_{2}$ en el suelo Malihue.

Table 4: Kd values for metsulfuron - methyl and triasulfuron caused by different doses of liming with $\mathrm{Ca}(\mathrm{OH})_{2}$ in Malihue soil.

\begin{tabular}{lcccc}
\hline \multirow{2}{*}{ Herbicidas } & \multicolumn{4}{c}{ Dosis de $\mathbf{C a}(\mathbf{O H})_{2}$ (t ha $\left.^{-1}\right)$} \\
\cline { 2 - 5 } & 0 & 1,9 & 3,7 & 7,4 \\
\hline Metsulfuron - metil & 0,88 & 0,30 & 0,14 & 0,08 \\
Triasulfuron & 2,89 & 2,28 & 1,56 & 0,53 \\
\hline
\end{tabular}

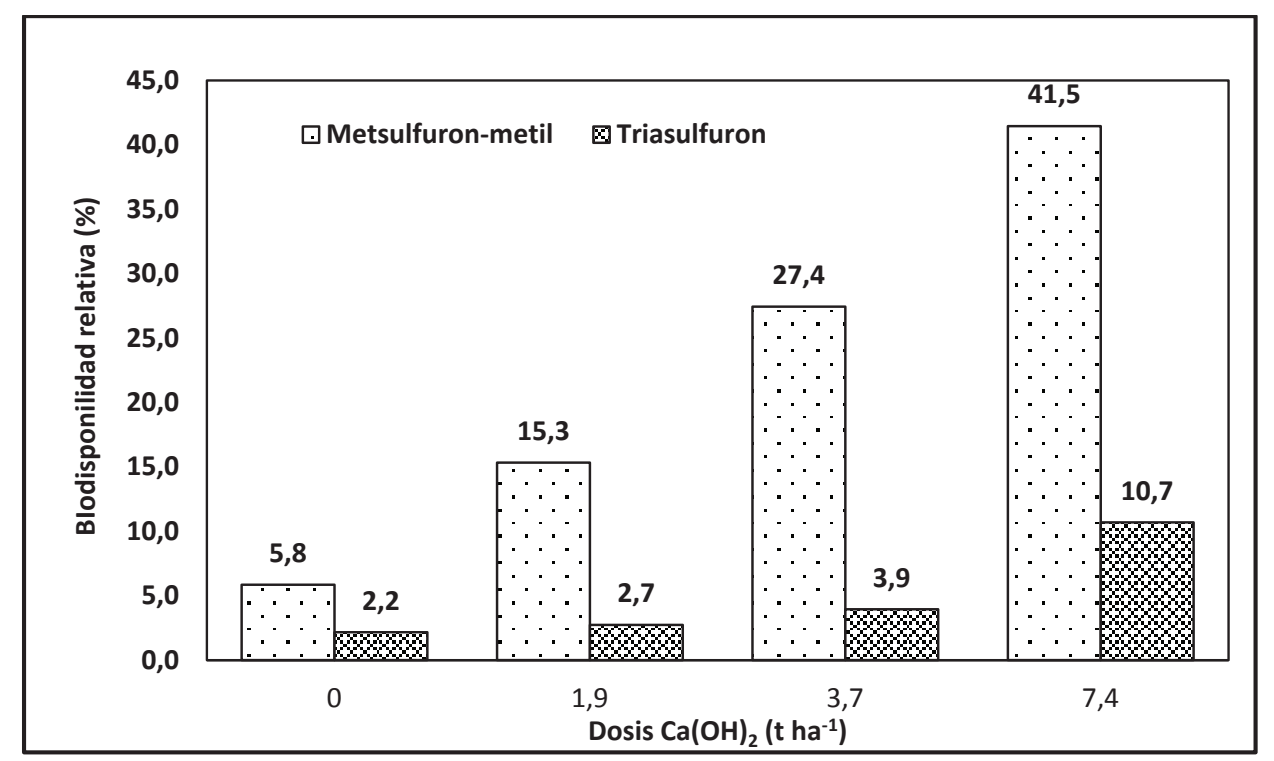

Figura 3: Biodisponibilidad relativa de metsulfuron-metil y triasulfuron bajo diferentes dosis de $\mathrm{Ca}(\mathrm{OH})_{2}$ en el suelo Malihue. Figure 3: Relative bioavailability of metsulfuron-methyl and triasulfuron under different doses of $\mathrm{Ca}(\mathrm{OH})_{2}$ in $\mathrm{Malihue} \mathrm{soil.}$ 
lo utilizado para ambos herbicidas se muestran en el Cuadro 5. Estos resultados indican que el modelo nolineal empleado se ajustó adecuadamente a los valores obtenidos, con coeficientes de determinación que variaron entre 0,93 y 1,0 (Cuadro 5). Los valores IC $_{50}$ disminuyeron a medida que aumentó la dosis de encalado. El control de cuarzo presentó el valor de $\mathrm{IC}_{50}$ más bajo entre todos los tratamientos empleados, necesitándose una menor dosis de aplicación para alcanzar el $50 \%$ del daño a nivel de raíz en ambos productos. Esto confirmaría la naturaleza no adsortiva del cuarzo. Por otra parte, al igual que en el suelo Malihue, en este suelo el análisis gráfico de la curva del testigo cuarzo (Figuras 4 y 5) confirman la mayor sensibilidad del

Cuadro 5: Coeficientes de regresión y determinación del modelo de curvas de respuesta utilizados para metsulfuron-metil y triasulfuron bajo distintas dosis de encalado en el suelo Fresia.

Table 5: Regression and determination coefficients of the response curve model used for metsulfuron-methyl and triasulfuron under different doses of liming in Fresia soil.

\begin{tabular}{|c|c|c|c|c|c|c|}
\hline \multirow[b]{2}{*}{ Herbicida } & \multirow[b]{2}{*}{ Coeficientes $(*)$} & \multicolumn{5}{|c|}{ Dosis de $\mathrm{Ca}(\mathrm{OH})_{2}$ tha $^{-1}$} \\
\hline & & $\mathbf{0}$ & 1,9 & 3,7 & 7,4 & Cuarzo \\
\hline \multirow{5}{*}{ Metsulfuron-metil } & $\mathrm{m}\left(\mathrm{IC}_{50}\right)$ & 10,16 & 9,29 & 8,64 & 8,51 & 7,30 \\
\hline & a & 40,48 & 37,90 & 25,66 & 15,46 & 21,66 \\
\hline & c & 59,52 & 62,10 & 74,34 & 84,54 & 78,34 \\
\hline & $\mathrm{b}$ & 1,52 & 0,82 & 1,09 & 0,71 & 0,68 \\
\hline & $r^{2}$ & 0,96 & 0,93 & 0,98 & 0,99 & 0,99 \\
\hline \multirow{5}{*}{ Triasulfuron } & $\mathrm{m}\left(\mathrm{IC}_{50}\right)$ & 9,53 & 9,01 & 8,41 & 8,12 & 5,69 \\
\hline & $\mathrm{a}$ & 26,65 & 23,75 & 19,42 & 11,30 & 18,23 \\
\hline & c & 73,35 & 76,25 & 80,58 & 88,70 & 81,77 \\
\hline & $\mathrm{b}$ & 1,57 & 1,06 & 0,65 & 0,50 & 0,76 \\
\hline & $r^{2}$ & 0,97 & 0,99 & 0,96 & 1,00 & 0,99 \\
\hline
\end{tabular}

$\left(^{*}\right) r^{2}$ : coeficiente de determinación; $\mathrm{IC}_{50}$ : Concentración de herbicida que inhibe el 50\% del crecimiento radical.

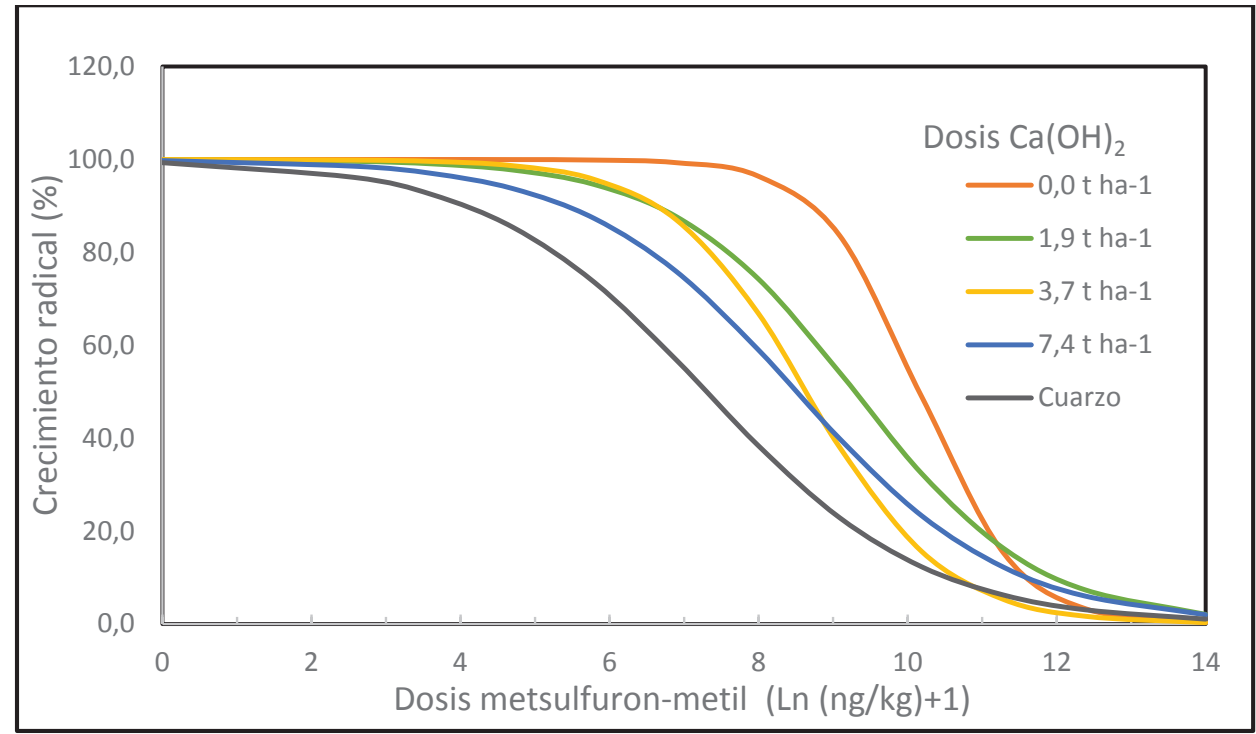

Figura 4: Curva respuesta para metsulfuron-metil bajo diferentes dosis de $\mathrm{Ca}(\mathrm{OH})_{2}$ en cuarzo y en el suelo Fresia.

Figure 4: Response curves for metsulfuron-methyl under different doses of $\mathrm{Ca}(\mathrm{OH})_{2}$ in quartz and in Fresia soil. 
sistema radical de la planta indicadora a las diferentes dosis de ambos herbicidas, presentando una mayor toxicidad de estos productos al comparar este testigo con los tratamientos con suelo, fundamentalmente por la nula adsorción que presentaría el cuarzo, encontrándose completamente disponible en la solución del suelo.

En el Cuadro 6 se presentan la comparación de los $\mathrm{IC}_{50}$ determinados para todos los tratamientos en el suelo Fresia. En general se observó que, para ambos herbicidas, los valores de $\mathrm{IC}_{50}$ obtenidos en el cuarzo fueron significativamente inferiores a los presentados en los tratamientos con suelo, ratificando nuevamente el efecto inerte del sustrato testigo. Los valores de $\mathrm{IC}_{50}$ para metsulfuron-metil fueron superiores a los obtenidos para triasulfuron, demostrando un mayor efecto tóxico de este último herbicida a menores dosis. Al igual que en el suelo Malihue, existió una relación inversa entre el valor del $\mathrm{IC}_{50}$ y la dosis de cal aplicada. Sin embargo, en ambos herbicidas solo existieron diferencias significativas entre los encalados $0,1,9$ y $3,7 \mathrm{t} \mathrm{ha}^{-1} \mathrm{y}$ no se detectaron diferencias entre $3,7 \mathrm{t} \mathrm{ha}^{-1}$ y $7,4 \mathrm{t} \mathrm{ha}^{-1}$ (Cuadro 6). Esto se explicaría porque al utilizar las dosis más altas de encalado se habrían alcanzado niveles de $\mathrm{pH}$ menores en el suelo Malihue $(5,72$ y 6,18$)$ que en el suelo Fresia $(6,66$ y 7,22), no existiendo variaciones significativas en la carga electrostática en este último suelo.

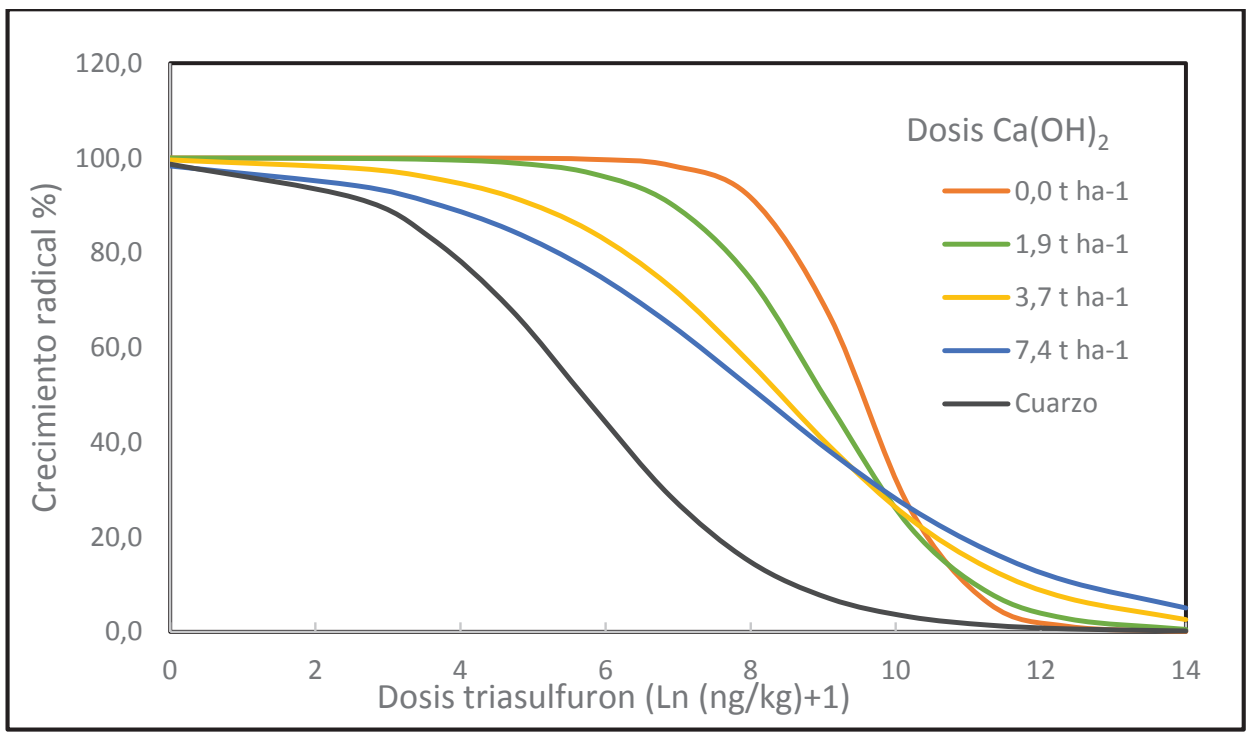

Figura 5: Curva respuesta para triasulfuron bajo diferentes dosis de $\mathrm{Ca}(\mathrm{OH})_{2}$ en cuarzo y en el suelo Fresia.

Figure 5: Response curves for metsulfuron under different doses of $\mathrm{Ca}(\mathrm{OH})_{2}$ in quartz and in Fresia soil.

Cuadro 6: Valores del $\mathrm{IC}_{50}$ y de concentración de metsulfuron-metil y triasulfuron con diferentes dosis de encalado en el suelo Fresia.

Table 6: $\quad \mathrm{IC}_{50}$ and concentration values of metsulfuron-methyl and triasulfuron with different doses of liming in Fresia soil.

\begin{tabular}{|c|c|c|c|c|c|}
\hline \multirow[b]{2}{*}{ Herbicida (unidad) } & \multicolumn{4}{|c|}{ Dosis de Ca(OH $)_{2}$ t ha $^{-1}$} & \multirow[b]{2}{*}{ Cuarzo } \\
\hline & $\mathbf{0}$ & 1,9 & 3,7 & 7,4 & \\
\hline \multicolumn{6}{|l|}{ Metsulfuron-metil IC ${ }_{50}$} \\
\hline$\left(\operatorname{Ln}\left(n g k^{-1}+1\right)\right)$ & $10,17 a^{*}$ & $9,30 \mathbf{b}$ & $8,64 \mathbf{c}$ & $8,52 \mathrm{c}$ & $7,31 \mathbf{d}$ \\
\hline (ng kgs.s. ${ }^{-1}$ ) & 25.977 & 10.880 & 5.667 & 5.006 & 1.491 \\
\hline$\left(\mathrm{g} \mathrm{ha}^{-1}\right)$ & 18 & 8 & 4 & 4 & 1 \\
\hline \multicolumn{6}{|l|}{ Triasulfuron $\mathrm{IC}_{50}$} \\
\hline$\left(\operatorname{Ln}\left(\mathrm{ng} \mathrm{kg}^{-1}+1\right)\right)$ & $9,53 \mathbf{a}$ & $9,01 \mathbf{b}$ & $8,40 \mathrm{c}$ & $8,12 \mathbf{c}$ & $5,69 \mathbf{d}$ \\
\hline (ng kgs.s. ${ }^{-1}$ ) & 13.783 & 8.204 & 4.480 & 3.368 & 296 \\
\hline$\left(\mathrm{g} \mathrm{ha}^{-1}\right)$ & 10 & 6 & 3 & 2 & 0,2 \\
\hline
\end{tabular}

* = Letras diferentes en la fila indican diferencias significativas $(\mathrm{p}<0,05)$ por el test de Tukey 
En este suelo, al igual que en el suelo Malihue, el incremento del valor de $\mathrm{pH}$ del suelo causado por el aumento de las dosis de cal redujo la proporción adsorbida de ambos herbicidas en los coloides del suelo. Al comparar la diferencia entre los valores de concentración equivalente de $\mathrm{IC}_{50}$ en el tratamiento con encalado menos el $\mathrm{IC}_{50}$ del tratamiento testigo cuarzo (Cuadro 6), se determinó que cuantitativamente la adsorción de metsulfuron-metil en el tratamiento sin cal $(\mathrm{pH}=5,55)$ alcanzó un total de $24.486 \mathrm{ng} \mathrm{kg}^{-1}$ y $3.515 \mathrm{ng}$ $\mathrm{kg}^{-1}$ con la aplicación de 7,4 t ha ${ }^{-1}$ de cal $(\mathrm{pH}=7,22)$, significando una reducción de adsorción relativa de $94,3 \%$ a $70,2 \%$, respectivamente. La adsorción de triasulfuron fue cuantitativamente inferior a metsulfuronmetil, presentando valores que variaron de $13.487 \mathrm{ng}$ $\mathrm{kg}^{-1}$ en el tratamiento testigo cuarzo a $3.072 \mathrm{ng} \mathrm{kg}^{-1}$ en el tratamiento con la máxima dosis de cal, pero mayor en términos relativos, dado que la adsorción se redujo de $97,9 \%$ a $91,2 \%$, respectivamente.

Los valores de biodisponibilidad relativa en la solución del suelo obtenidos para ambos herbicidas se muestran en la Figura 6, en ellos se observa que al comparar el suelo Fresia con una incorporación equivalente a $7,4 \mathrm{t}$ de cal ha-1 $(\mathrm{pH}=7,22)$ con el suelo sin aplicación de cal $(\mathrm{pH}=5,25)$, se acrecentó la biodisponibilidad de metsulfuron-metil de $5,7 \%$ a $29,8 \%$, incremento inferior al observado en el suelo Malihue. En el caso de triasulfuron existió la misma tendencia, solo que el incremento fue menor aumentando su biodisponibilidad solo de $2,1 \%$ a $8,8 \%$, valores similares a los presentados en el suelo Malihue. El coeficiente de adsorción
(Kd) disminuyó a medida que se usó mayor dosis de encalado, variado entre 0,90 a 0,13 en metsulfuron metil y entre 2,91 a 0,66 para triasulfuron (Cuadro 7). Esto comprobaría como la cantidad de herbicida presente en la solución del suelo aumenta con relación a la cantidad adsorbida en el suelo en la medida que sube el pH del suelo con el aumento de la dosis de encalado. Considerando que ambos herbicidas son aniónicos y que tienen una tasa de hidrólisis $\mathrm{pH}$ dependiente, estos resultados estarían ratificando el hecho que aplicaciones de cal aumentarían la proporción de herbicida en la solución, como consecuencia de una menor ionización por una redución de su tasa de hidrólisis al producirse un aumento de $\mathrm{pH}$.

Cuadro 7: Valores de Kd para metsulfuron - metil y triasulfuron por efecto de diferentes dosis de encalado con $\mathrm{Ca}(\mathrm{OH})_{2}$ en el suelo Fresia.

Table 7: Kd values for metsulfuron - methyl and triasulfuron caused by different doses of liming with $\mathrm{Ca}(\mathrm{OH})_{2}$ in Fresia soil.

\begin{tabular}{lcccc}
\hline \multirow{2}{*}{ Herbicidas } & \multicolumn{4}{c}{ Dosis de Ca(OH) ${ }_{2}\left(\mathbf{t ~ h a}^{-1}\right)$} \\
\cline { 2 - 5 } & $\mathbf{0}$ & $\mathbf{1 , 9}$ & $\mathbf{3 , 7}$ & $\mathbf{7 , 4}$ \\
\hline Metsulfuron - metil & 0,90 & 0,34 & 0,15 & 0,13 \\
Triasulfuron & 2,91 & 1,71 & 0,90 & 0,66 \\
\hline
\end{tabular}

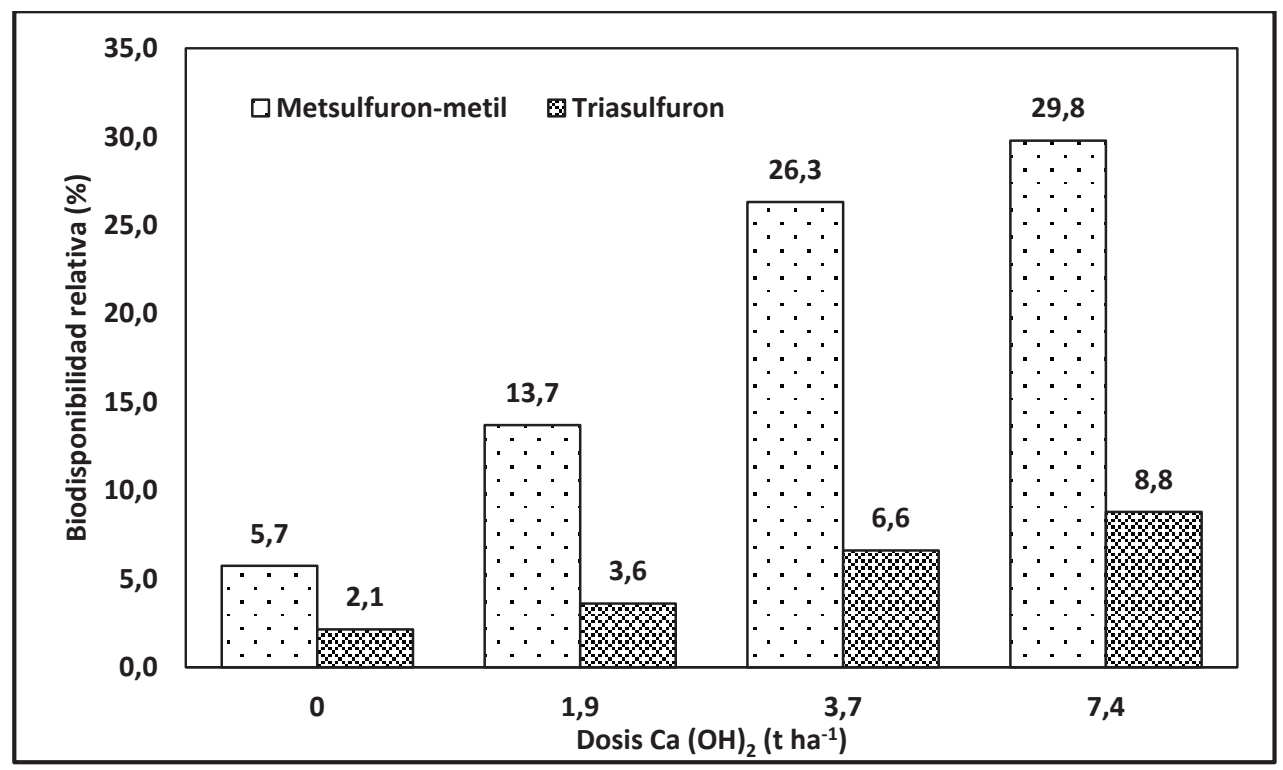

Figura 6: Biodisponibilidad relativa de metsulfuron-metil y triasulfuron bajo diferentes dosis de $\mathrm{Ca}(\mathrm{OH})_{2}$ en el suelo Fresia. Figure 6: Relative bioavailability of metsulfuron-methyl and triasulfuron under different doses of $\mathrm{Ca}(\mathrm{OH})_{2}$ in Fresia soil. 


\section{DISCUSIÓN}

La capacidad tampón de pH varía entre tipos de suelos y dentro de un mismo suelo, dependiendo de su capacidad de intercambiar catiónes, es decir, depende del tipo y contenido de arcilla predominante y el contenido de materia orgánica del suelo (Halvin et al., 1999; Pinochet et al., 2005). Ambos suelos presentaron diferencias en su capacidad tampón de pH, observándose que el suelo Malihue, comparado con el suelo Fresia, requirió una mayor dosis de encalado para incrementar en una unidad el pH del suelo, resultados que fueron concordantes con las diferencias observadas en los valores de $\mathrm{IC}_{50}$. Para el suelo Malihue los valores de $\mathrm{IC}_{50}$ fueron superiores a los reportados por la literatura para herbicidas del grupo de las sulfonilureas (Günther et al., 1989; Stork y Hannah, 1996) con metodologías similares, situación que puede deberse a la naturaleza adsortiva del suelo, el cual corresponde a un suelo trumao que se caracteriza por tener arcillas reactivas (alofán) y alto contenido de materia orgánica (Luzio, 2010). El incremento de la dosis de encalado trae consigo una mayor capacidad de intercambio de cationes, lo que aumenta las cargas negativas en la superficie coloidal (Rahman et al., 1996) provocando un fenómeno de repulsión electrostática de las moléculas de metsulfuron-metil hacia la solución del suelo. Ren et al. (2011), determinaron que los suelos pueden ser más susceptibles a pérdidas por lixiviación de sulfonilureas si las enmiendas aplicadas aumentan el pH del suelo. Por lo tanto, en suelos derivados de cenizas volcánicas la aplicación de enmiendas orgánicas o calcáreas que incrementen el pH permitirán disminuir la retención del herbicida dado por el aumento en la capacidad de intercambio catiónico desde la superficie cargada (Kotoula-Syka et al., 1993). De esta manera, el suelo Malihue bajo condición de pH básico necesita una menor dosis de herbicida para lograr el 50\% de la inhibición del crecimiento radical.

En el suelo Fresia las diferencias entre la concentración que inhibe el $50 \%$ el sistema radical de la planta indicadora en el cuarzo y en el tratamiento con mayor encalado (Cuadro 3 y 5) es mayor que en el suelo Malihue, en ambos herbicidas; esto podría explicarse por el nivel de fósforo inicial mayor en el suelo Fresia, lo que tiende a disminuir los sitios activos de adsorción por acción de los iones fosfato presentes en el suelo a pesar de su capacidad de adsorción dada por la fracción coloidal predominante (Cáceres et al., 2010b). Esto derivaría en una mayor biodisponibilidad del herbicida por un aumento de la repulsión aniónica de sus moléculas cargadas negativamente (Cuadro 6). Por otro lado, en el suelo Fresia las curvas de las dosis más altas de encalado $\left(3,7\right.$ y $\left.7,4 \mathrm{t} \mathrm{ha}^{-1}\right)$ tienden a estar más cerca del control debido a que alcanzaron pH más altos $(6,66$ y $7,22)$, lo cual influye en la carga variable del suelo, pro- vocando un aumento de las cargas negativas (Cáceres et al., 2010a; Singh y Singh, 2012). Este fenómeno puede estar dado por la saturación de las cargas electrostáticas del suelo y las diferencias de adsorción entre el control y las dosis más altas de encalado, debido probablemente a otro tipo de adsorciones específicas. Por lo tanto, esto indicaría que el herbicida metsulfuron-metil en condiciones naturales presenta una alta adsorción en el suelo, y que logra quedar rápidamente disponible a medida que el encalado se incrementa en el suelo. Según Günther et al. (1989), los IC I0 $_{50}$ de metsulfuron-metil y triasulfuron son mayores en suelos con alto contenido de materia orgánica.

La clara tendencia observada a la disminución de la adsorción de herbicida y el aumento de su biodisponibilidad en solución a medida que incrementa el pH coincide por lo reportado por Ren et al. (2011), situación que podría mejorar la eficacia del herbicida para controlar malezas (Fuentes et al., 2005) o simplemente puede ser lixiviado a capas más profundas de suelo o al agua subterránea (Cáceres et al., 2010b). En el suelo Fresia, la adsorción de ambos herbicidas se estabilizaría a pH sobre 6,66. Este resultado podría ser explicado por el hecho que cuando el pH supera 6,8, por debajo de este valor las sulfonilureas se encuentran bajo la forma no disociada con carga neutral a levemente positiva, lo suficiente para estar adsorbida a las arcillas y materia orgánica del suelo cargados negativamente. Por el contrario con valores superiores a 6,8 sus moléculas liberan protones $\left(\mathrm{H}^{+}\right)$consecuentemente se cargan negativamente y son repelidas tanto por los minerales como la materia orgánica del suelo (Walker et al., 1989) o, estando disponibles para la absorción de las plantas por un mayor periodo mayor de tiempo es decir incrementan su residualidad.

Los pH alcanzados en ambos suelos con encalados de 7,4 t ha ${ }^{-1}$ de $\mathrm{Ca}(\mathrm{OH})_{2}$ son muy altos especialmente para el suelo Fresia, lo cual provocaría una gran repulsión aniónica. Esto reafirma la posibilidad de que existan adsorciones específicas o competencia adsortivas con otros iones tales como los fosfatos o complejos orgánicos provenientes de la materia orgánica del suelo. Por lo tanto, una alta carga variable tanto de las arcillas como de la materia orgánica del suelo, provocaría un aumento de la biodisponibilidad de herbicida al aumentar el pH con dosis crecientes de enmiendas calcáreas, lo que es importante ya que un encalado ayudaría a mejorar la eficiencia de ambos herbicidas.

La estructura química molecular de las sulfonilureas (metsulfuron-metil y triasulfuron) no difieren mucho una de otra (Anderson et al., 2010), pero la tendencia de adsorción en el suelo, es que el metsulfuronmetil este más biodisponible que el triasulfuron en el suelo en ambos suelos. Por otro lado, la disminución del Kd en ambos herbicidas en los dos suelos estudiados, indica que el metsulfuron-metil se adsorbe menos 
que el triasulfuron (Kotoula-Syka et al., 1993). Esto se explica porque la constante de disociación (pKa) del metsulfuron-metil es menor que el triasulfuron, por lo tanto al mismo $\mathrm{pH}$ el metsulfuron-metil se encuentra más ionizado y mayormente repelido por las cargas negativas del suelo (Walker et al., 1989).

\section{CONCLUSIONES}

El requerimiento de cal para elevar el valor de $\mathrm{pH}$ en el suelo Fresia (Paleohumult) fue menor que en el suelo Malihue (Hapludand), ya que al utilizar las dosis más altas de encalado se alcanzaron los niveles de $\mathrm{pH}$ menores en el suelo Malihue que en el suelo Fresia. Los valores de $\mathrm{IC}_{50}$ para cada herbicida fueron similares en ambos suelos, sin embargo los valores de metsulfuronmetil fueron siempre superiores a los obtenidos para triasulfuron demostrando el mayor efecto tóxico de triasulfuron a menores dosis. La adsorción electrostática del metsulfuron-metil y el triasulfuron disminuye a medida que aumenta la dosis de encalado con $\mathrm{Ca}(\mathrm{OH})_{2}$, en ambos suelos estudiados, aumentando su biodisponibilidad en la solución suelo. La adsorción en el suelo Fresia tendió a estabilizarse a pH 6,66 lograda con una encaladura equivalente a $3,7 \mathrm{t} \mathrm{ha}^{-1}$, estabilización que no se observó en el suelo Malihue. La adsorción de triasulfuron con el incremento de las aplicaciones de cal fue inferior a metsulfuron-metil en términos cuantitativos, pero mayor en términos relativos. En el caso del triasulfuron, en general, la biodisponibilidad relativa fue menor que para metsulfuron-metil, en ambos suelos, aumentando con el incremento de la dosis de aplicación de cal. Estos resultados se corroboraron al examinar los coeficientes de adsorción (Kd).

\section{REFERENCIAS}

ABDULLAH, A., SINNAKKANNU, S., TAHIR, N. 2001. Adsorption-desorption behaviour of metsulfuron-methyl in selected Malaysian agriculture. Fresenius Environment Bulletin 10: 94-97.

ANDERSON, D. M., CAROLAN, V. A., CROSLAND, S., SHARPLES, K. R., CLENCH, M. R. 2010. Examination of the translocation of sulfonylurea herbicides in sunflower plants by matrix-assisted laser desorption/ionisation mass spectrometry imaging. Rapid Communications in Mass Spectrometry 24(22): 3309-3319.

BLACKLOW, W., PHELOUNG, P. 1992. Sulfonylurea herbicides applied to acid sand soils: Movement, persistence and activity within the growing season. Australian Journal of Agricultural Research 43: 1205-1216.

BROWN, H. 1990. Mode of action, crop selectivity, and soil relation of sulphonylurea herbicides. Pesticide Science 29: 263-281.
CÁCERES, L., ESCUDEY, M., FUENTES, E., BÁEZ, M. E. 2010a. Modeling the sorption kinetic of metsulfuron-methyl on Andisols and Ultisols volcanic ash-derived soils: kinetics parameters and solute transport mechanisms. Journal of Hazardous Materials 179(1): 795-803.

CÁCERES, L., FUENTES, R., ESCUDEY, M., FUENTES, E., BÁEZ, M. E. 2010b. Metsulfuron-methyl sorption/desorption behavior on volcanic ash-derived soils. Effect of phosphate and $\mathrm{pH}$. Journal of Agricultural and Food Chemistry 58(11): 6864-6869.

FUENTES, R., JEREZ, G., PINOCHET, D. 2005. Efecto de enmiendas calcáreas sobre la eficacia de metsulfuronmetil en el control de malezas en trigo. Revista de la Ciencia del Suelo y Nutrición Vegetal (1): 1-7.

GÜNTHER, P., RAHMAN, A., PESTEMER, W. 1989. Quantitative bioassays for determining residues and availability to plants of sulphonylurea herbicides. Weed Research 29: 141-146.

HAVLIN, J. L., BEATON, J. D., TISDALE, S. L., NELSON, W. L. 1999. Soil fertility and fertilizers: An introduction to nutrient management. Prentice Hall, New Jersey, USA.

HERNÁNDEZ-SEVILLANO, E., VILLARROYA, M., ALONSOPRADOS, J. L., GARCÍA-BAUDÍN, J. M. 2001. Bioassay to Detect MON-37500 and Triasulfuron Residues in Soils 1. Weed Technology 15(3): 447-452.

ISMAIL, B. S., OOI, K. E. 2012. Adsorption, desorption and mobility of metsulfuron-methyl in soils of the oil palm agroecosystem in Malaysia. Journal of Environmental Biology 33(3): 573.

KOTOULA-SYKA, E. , ELEFTHEROHORINOS, I.G., GAGIANAS, A.A, SFICAS, A.G. 1993. Phytotoxicity and persistence of chlorsulfuron, metsulfuron-methyl, triasulfuron and tribenuron-methyl in three soils. Weed Research 33: 355-367.

LUZIO, W., CASANOVA, M. 2006. Avances en el conocimiento de los suelos de Chile. Editorial Universidad de Chile Servicio Agrícola y Ganadero (SAG). Santiago, Chile.

LUZIO, W. 2010. Suelos de Chile. Impreso Maval, Universidad de Chile, Santiago.

MORA, M.L., DEMANET, R. 1999. Uso de enmiendas calcáreas en suelos acidificados. Frontera Agrícola 5: 43-58.

PINOCHET, D., RAMÍREZ, F., SUÁREZ, D. 2005. Variación de la capacita tampón en suelos derivados de cenizas volcánicas. Agricultura Técnica 65(1): 55-64.

RAHMAN, A., JAMES, T.K., CORNWELL, M.J. 1996. Influence of lime on activity and persistence of tribenuron and thifensulfuron in soil. $49^{\text {th }}$ Conference Proceedings of the New Zealand Plant Protection. pp. 202-206.

REN, W., WANG, M., ZHOU, Q. 2011. Adsorption characteris- 
tics and influencing factors of chlorimuron-ethyl in two typical chinesse soils. Soil Science Society of America Journal 75(4): 1394-1401.

SARMAH, A. K., SABADIE, J. 2002. Hydrolysis of sulfonylurea herbicides in soils and aqueous solutions: a review. Journal of Agricultural and Food Chemistry 50(22): 6253-6265.

SEKUTOWSKI, T. 2011. Application of bioassays in studies on phytotoxic herbicide residues in the soil environment. In: Herbicides and Environment. Kortekamp, A. (Ed.) InTech. Open Access Publisher. pp. 253-272.

SINGH, S. B., KULSHRESTHA, G. 2006. Soil persistence of triasulfuron herbicide as affected by biotic and abiotic factors. Journal of Environmental Science and Health Part
B 41(5): 635-645.

SINGH, N., SINGH, S. B. 2012. Sorption-desorption behavior of metsulfuron-methyl and sulfosulfuron in soils. Journal of Environmental Science and Health, Part B 47(3): 168-174.

STORK, P., HANNAH, M. 1996. A bioassay method for formulation testing and reside studies of sulfonylurea and sulfonanylide herbicides. Weed Research 36: 271-281.

WALKER, A., COTTERILL, E., WELCH, S. 1989. Adsorption and degradation of chlorsulfuron and metsulfuron - metil in soils from different depths. Weed Research 29: 281-383.

WALKER, A., WELCH, S. 1989. The relative movement and persistence in soils of chlorsulfuron, metsulfuron- metil and triasulfuron. Weed Research 29: 375-383. 